\title{
Ultra-wideband Channel Modeling for Hurricanes
}

\author{
Wahab Khawaja*, Ismail Guvenc*, and Arindam Chowdhury ${ }^{\dagger}$ \\ ${ }^{\dagger}$ Department of Electrical and Computer Engineering, Florida International University, Miami, FL \\ ${ }^{*}$ Department of Electrical and Computer Engineering, North Carolina State University, Raleigh, NC \\ Email: \{wkhawaj, iguvenc\}@ncsu.edu, \{chowdhur\}@fiu.edu
}

\begin{abstract}
ПAbstract-Maintaining communications during major hurricanes is critically important for public safety operations by first responders. This requires accurate knowledge of the propagation channel during hurricane conditions. In this work, we have carried out ultra-wideband (UWB) channel measurements during hurricane conditions ranging from Category-1 to Category4 , generated at the Wall of Wind (WoW) facility of Florida International University (FIU). Time Domain P410 radios are used for channel measurements. From the empirical data analysis in time domain, we developed a UWB statistical broadband channel model for hurricanes. In particular, we characterize the effects of rain and wind speed on large scale and small scale UWB propagation parameters.
\end{abstract}

Index Terms - Channel measurement, channel modeling, hurricane, Ultrawideband (UWB).

\section{INTRODUCTION}

Hurricanes are one of most destructive forces in nature where wind speeds exceeding $74 \mathrm{mph}$ and heavy rainfall are observed. An estimated 18 hurricanes of different categories hit United States every decade on the average [1]. Hurricanes can lead to difficulties in existing communication links as the design of general radio transceivers do not take into account such severe conditions. This can make the rescue and relief operations during those conditions extremely challenging. Other sensitive operations such as air traffic control and military communications are also affected in such conditions. With the recent use of autonomous modes of transportation in air, sea and ground, a reliable communication link in all weather conditions is paramount.

Ultra-wideband (UWB) radio signals with their large bandwidth, high data rates, and minimum interference to existing communication links are used in numerous applications. These applications include radars for search, rescue and imaging in the disaster hit areas. A reliable propagation channel model in these conditions such as hurricanes can help in efficient rescue operations. There are UWB channel models available in the literature for indoor and outdoor environments [2], [3]. However, to our best knowledge, there is no study available to characterize UWB channels in high wind and rain conditions. In [4]-[9] weather effects on communication channel at different frequencies are discussed in controlled and outdoor environments with different foliage concentrations. The wind speeds and rain intensities considered in these studies are much lower than those encountered in a hurricane.

In this work, we have carried out channel sounding of UWB radio signals in hurricanes generated from Category-1 to Category-4 based on Saffir-Simpson hurricane scale [10]

This work has been supported in part by the NSF grants AST-1443999 and CNS-1453678, and W. Khawaja has been supported via a Fulbright scholarship.

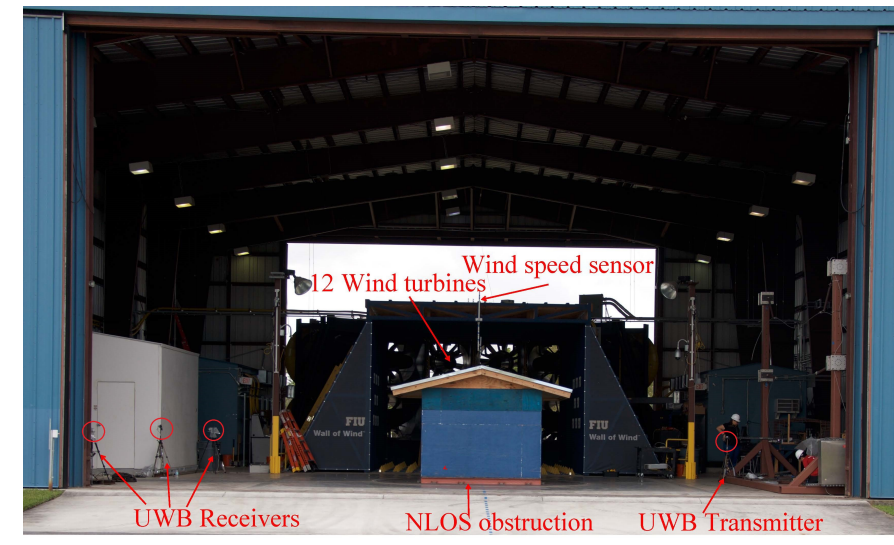

Fig. 1. UWB channel measurement setup at Florida International University, Wall of Wind facility, Miami, FL.

at Wall of Wind (WoW) facility in Florida International University (FIU) with the set up shown in Fig. 11. Time domain P410 radios are used for pulse based channel sounding. The frequency range of operation is $3.1 \mathrm{GHz}-5.3 \mathrm{GHz}$. The empirical analysis of data in time domain is carried out in order to build a statistical channel model for line-of-sight (LOS), and none-line-of-sight (NLOS) propagation paths in different scenarios during a hurricane. The statistical channel model is found to closely fit the empirical data.

\section{UWB Channel SOUNDING AT FIU WoW}

In this section we summarize the experimental set up at FIU WoW. Special measures were taken in order to protect the communication and data storage equipment from hurricane effects and relaying radio controls from a safe point.

\section{A. Channel Measurements with UWB P410 Radios}

Time Domain P410 radios in bi-static mode are used in channel measurements due to their ease of setting up and efficiently measuring the channel response in a concise space. The operating frequency range for the experiment is $3.1 \mathrm{GHz}$ - 5.3 GHz. The transmitted power from the radio was limited to $-14.5 \mathrm{dBm}$ due to $\mathrm{FCC}$ requirements [11]. The radios are configured to send pulses at a rate of $10.1 \mathrm{MHz}$ and for a scan duration of $100 \mathrm{~ns}$. The width of each pulse is $1 \mathrm{~ns}$. A pseudo-random (PR) coded pulse train sequence is used to provide synchronization between the transmitter and receivers eliminating the requirement for a physical connection between the transmitter and the receiver. The PR encoded pulses in the acquisition preamble of the transmitted packet is used to detect and lock the transmitted data at a given receiver. The synchronization clock information is sent from the transmitter 

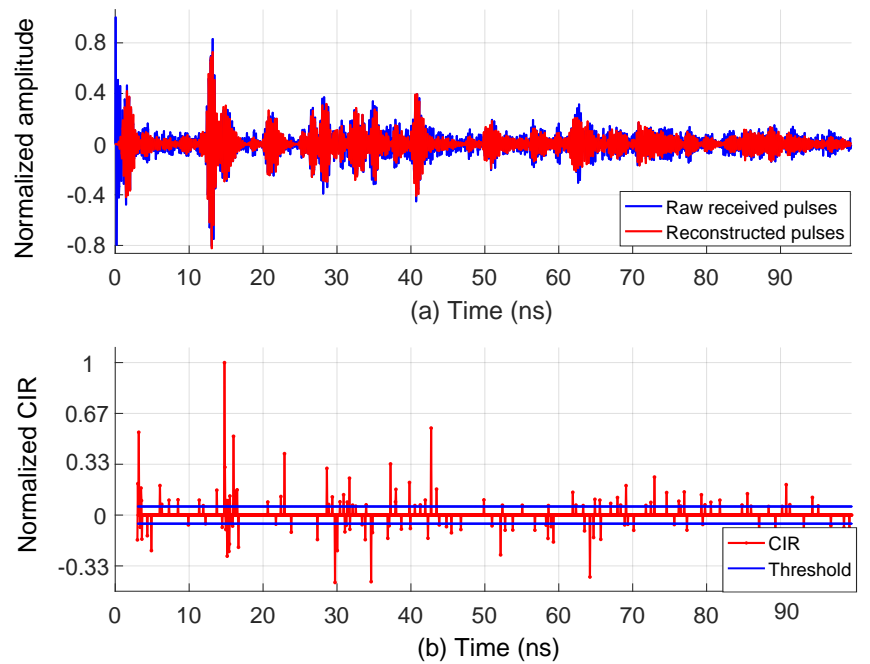

Fig. 2. (a) Normalized amplitude of pulses with respect to time for a given scan (blue represents received pulses and red represents reconstructed pulses), (b) Normalized CIR with respect to time for a given scan.

to receivers through transmitted packets. The rake receiver [12] is used to collect the energy in the transmitted waveform at a sampling resolution of $61 \mathrm{ps}$ at the receiving radios.

UWB BroadSpec planar elliptical dipole antennas are used in the experiment. The antenna pattern is omni-directional in azimuth direction and doughnut shaped in the vertical direction with gain of $3 \mathrm{dBi}$. Antennas are placed such that bore-sight of transmitter and receiver antennas face each other giving higher gains [13]. The raw received and reconstructed pulses are shown in Fig. 2(a). In order to obtain reconstructed pulses, the template waveform is convolved with the channel impulse response (CIR) given in Fig. 2 (b). The CIR is obtained by deconvolving the received pulses with the template waveform using CLEAN algorithm. The horizontal blue line in Fig. 2. b) is the amplitude threshold set at $10 \%$ of the input signal. We have used raw received pulse data in our analysis.

\section{B. Hurricane Generation Setup}

At the FIU WoW, 12 powerful fans are used to generate hurricanes from Category-1 to Category-5 [10]. We limited our experiments up to Category-4 hurricane due to limitations of the communication and relaying equipment. Rain is produced through water nozzles placed alongside the fans. The rain intensity in our experiment was set at $223.5 \mathrm{~mm} / \mathrm{h}$.

The wind pressure due to velocity and density of wind flow at a given spatial location is called pressure head. The density of wind will be considered to be constant along our observation length. From Fig. 3, the position P2 will be under lower pressure head as compared to P1 according to Bernoulli equation along a streamline [14]. This means that radios at P2 will be exposed to higher wind gusts as compared to at P1.

In case of wind driven rain (WDR), due to non-uniform motion of water droplets, a plausible assumption is the formation of large sized droplets due to smearing in to each other at higher wind velocities [15]. These large sized water droplets have higher likelihood to interfere with the electromagnetic (EM) waves. Due to large frequency band of the EM wave in the experiment, different frequency components will expe-

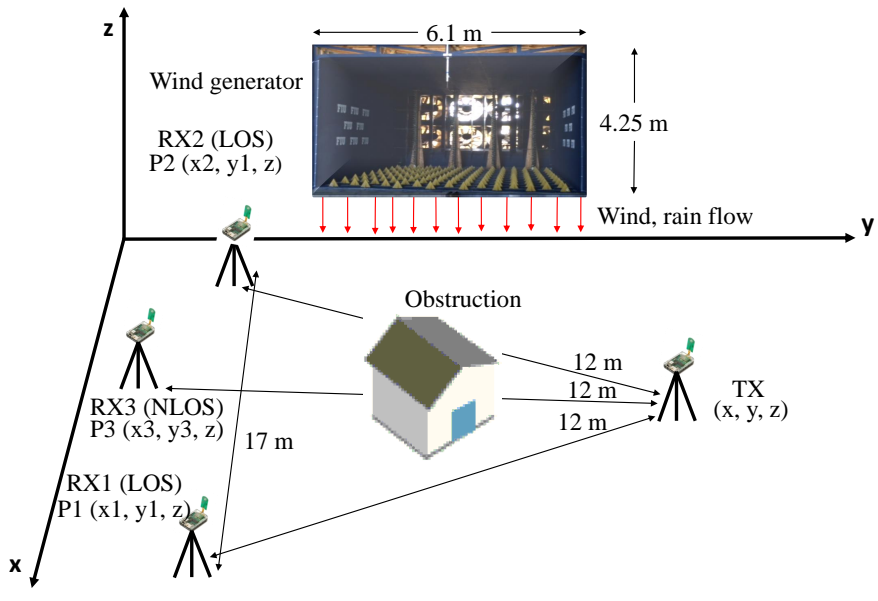

Fig. 3. Layout of UWB channel sounding scenario at FIU WoW.

rience different attenuation due to scattering, refraction and diffraction during rain.

Three receivers are placed at equal distance from the transmitter at $12 \mathrm{~m}$ as shown in Fig. 3. The LOS radios are at position $\mathrm{P} 1$ and $\mathrm{P} 2$, and a NLOS path is created by placing a wooden building structure of height $2.76 \mathrm{~m}$ between the transmitter and the receiver at position $\mathrm{P} 3$. The height of the transmitter and receivers is kept same at $1.5 \mathrm{~m}$. Two scenarios are studied for each receiver radio position labeled as $\mathrm{S} 1$ and $\mathrm{S} 2$. In $\mathrm{S} 1$, we have no rain, whereas in $\mathrm{S} 2$, we have rain. In both scenarios, the wind velocity is varied from $90 \mathrm{mph}$ to $140 \mathrm{mph}$ in six discrete steps. To reduce the impact of static objects in the environment, we subtracted the mean statistical CIR at each radio positions without hurricane conditions from the measured ones in hurricane.

\section{UWB CHANNEL MOdELING FOR HURRICANES}

In this section, a statistical channel model for UWB radio signals in the frequency band $3.1 \mathrm{GHz}-5.3 \mathrm{GHz}$ is developed for LOS and NLOS paths in hurricane based on the empirical data. We use modified Saleh Valenzuela (SV) channel model [16] for the representation of our UWB channel. The UWB channel in our analysis is considered to be linear time invariant with frequency selective fading.

\section{A. Multipath Components Analysis}

UWB channel can be characterized based on its multipath components (MPCs) obtained from its CIR:

$$
H(n)=\sum_{i=1}^{N} \sum_{l=1}^{L} a_{i, l} \exp \left(j \phi_{i, l}\right) \delta\left(n-\Gamma_{i}-\tau_{i, l}\right),
$$

where $H(n)$ is the discrete UWB channel response in time domain, $L$ is the total number of MPCs in the $i^{\text {th }}$ cluster, $N$ represents the total number of clusters during the scan interval, $a_{i, l}, \phi_{i, l}, \tau_{i, l}$ represent the amplitude, phase and delay of the $l^{\text {th }}$ MPC in the $i^{\text {th }}$ cluster, respectively, and $\Gamma_{i}$ is the delay of the $i^{\text {th }}$ cluster. The phase is a uniformly distributed random variable in the interval [ $\left[\begin{array}{ll}0 & 2 \pi\end{array}\right]$, thus it can be neglected. Power delay profile (PDP) obtained using CIR in the LOS case is shown in Fig. 4. The formation of major 


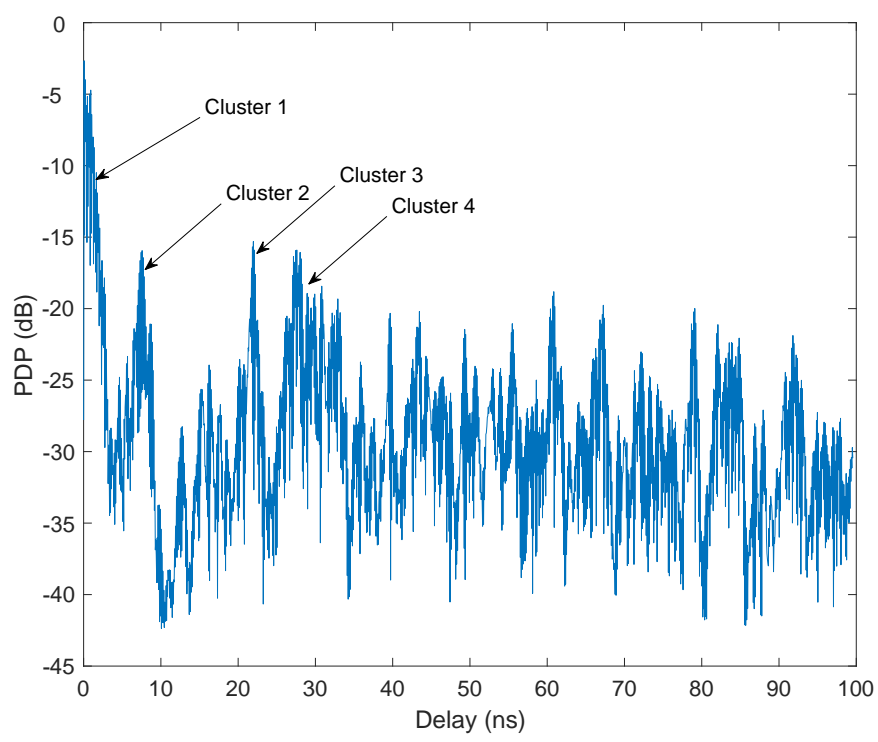

Fig. 4. Normalized PDP with respect to delay for a given scan.

clusters is due to reflection of the MPCs from objects of size comparable or larger than $13 \mathrm{~cm}$. We identify clusters in the PDP using our cluster identification algorithm that is based on the covariance likelihood of the samples of the PDP. The gain amplitudes of MPCs from measurements better fit to the lognormal distribution instead of Rayleigh distribution in the SV model. The amplitudes of channel coefficients can be represented as $a_{i, l}=e^{Z} \sim \mathcal{L N}\left(\mu_{\mathrm{a}_{\mathrm{i}, 1}}, \sigma_{\mathrm{a}}^{2}\right)$ where $Z$ is a normally distributed random variable with mean $\mu_{\mathrm{a}_{\mathrm{i}, 1}}$ and variance $\sigma_{\mathrm{a}}^{2}$, respectively.

The TOA of clusters and MPCs within each cluster follows two separate Poisson processes. For a given radio position if $\gamma$ and $\zeta$ represent the arrival rate of cluster and MPCs within each cluster, then we have [3], [16]:

$$
\begin{aligned}
p\left(\Gamma_{i} \mid \Gamma_{i-1}, \gamma\right) & =\gamma \exp \left(-\gamma\left(\Gamma_{i}-\Gamma_{i-1}\right)\right), \\
p\left(\tau_{i, l} \mid \tau_{i, l-1}, \zeta\right) & =\zeta \exp \left(-\zeta\left(\tau_{i, l}-\tau_{i, l-1}\right)\right) .
\end{aligned}
$$

If $\bar{\Gamma}$ and $\bar{\tau}$ represent the mean arrival time of clusters and MPCs within each cluster, respectively, then the effect of wind velocity and WDR can be given as

$$
\bar{\Gamma}=\left(1+c_{\mathrm{c}}\right) \bar{\Gamma}_{\mathrm{b}}, \quad \bar{\tau}=\left(1+c_{\mathrm{m}}\right) \bar{\tau}_{\mathrm{b}},
$$

where $0 \leq c_{\mathrm{c}}<1$ and $0 \leq c_{\mathrm{m}}<1$ are arbitrary constants that are dependent on the rain and pressure head, $c_{\mathrm{c}}>c_{\mathrm{m}}$, while $\bar{\Gamma}_{\mathrm{b}}$ and $\bar{\tau}_{\mathrm{b}}$ are the mean arrival time of clusters and MPCs within respective clusters for the base case without hurricane. The variation in the mean arrival time of cluster and MPCs within them is represented by two random variables $X_{\mathrm{c}} \sim$ $\mathcal{N}\left(0, \sigma_{\mathrm{c}}^{2}\right)$ and $X_{\mathrm{m}} \sim \mathcal{N}\left(0, \sigma_{\mathrm{m}}^{2}\right)$, respectively.

If the mean number of clusters at a given receiver radio position is represented by $\bar{N}$, then we have $\bar{N}=\left(1+\frac{c_{j} c_{\mathrm{p}}}{c_{r}}\right) \bar{N}_{\mathrm{b}}$ for $j=1,2,3, c_{j} \geq 0$ is a constant for given receiver radio position in hurricane, $c_{\mathrm{p}}>0$ and $c_{\mathrm{r}}>0$ are constants proportional to pressure head and rain, respectively, and $\bar{N}_{\mathrm{b}}$ is the mean number of clusters for the base case without hurricane. If $X_{\overline{\mathrm{N}}}$ is the random variable representing the variation in the mean number of clusters for a given radio position, then we have $X_{\overline{\mathrm{N}}} \sim \mathcal{N}\left(0, \sigma_{\overline{\mathrm{N}}}^{2}\right)$. The formation of clusters is dependent on the channel conditions e.g. during rain we have reduced reflectivity from objects and also EM waves experience higher dielectric constant and loss tangent of water as compared to air resulting in higher attenuation of MPCs and formation of fewer number of clusters.

\section{B. Power Delay Profile}

The PDP can help to characterize power distribution in a given channel as a function of propagation delay. We will consider the general case of non-overlapping clusters as basis for our analysis, where the PDP can be written as [3], [16].

$$
\begin{aligned}
P(n) & =\sum_{i=1}^{N} P_{\mathrm{mp}}^{i}(n) \exp \left(-\frac{\Gamma_{i}}{\Lambda}\right) \delta\left(n-\Gamma_{i}\right), \\
P_{\mathrm{mp}}^{i}(n) & =\sum_{l=1}^{L} E\left(a_{i, 1}^{2}\right) \exp \left(-\frac{\tau_{i, l}}{\lambda}\right) \delta\left(n-\tau_{i, l}\right),
\end{aligned}
$$

where $P(n)$ denote the PDP for $N$ clusters, $P_{\mathrm{mp}}^{i}(n)$ is the PDP for MPCs of $i$ th cluster, while $\Lambda$ and $\lambda$ are the power decay constants for inter-cluster and intra-cluster, respectively, and $E\left(a_{i, 1}^{2}\right)$ is the average power corresponding to first MPC of the $i_{\text {th }}$ cluster. The decay constant for inter-cluster case is larger than the intra-cluster case, i.e., $\Lambda>\lambda$. We can represent the variation in $P(n)$ and $P_{\mathrm{mp}}(n)$ from $\left.(5), 6\right)$ by two zero mean normally distributed random variables $X_{\mathrm{P}} \sim \mathcal{N}\left(0, \sigma_{\mathrm{P}}^{2}\right)$ and $X_{\mathrm{mp}} \sim \mathcal{N}\left(0, \sigma_{\mathrm{mp}}^{2}\right)$, respectively.

1) WDR effect on PDP: The PDP is effected by WDR due to phenomenon discussed in Section II-B. If $P^{\mathrm{nr}}(n)$ represents the PDP with no rain and $P^{\text {rn }}(n)$ represent the PDP in the presence of WDR then we have $P^{(\mathrm{rn})}(n)=\beta P^{(\mathrm{nr})}(n)+X_{\mathrm{R}}$, where $0<\beta<1$ is an attenuation constant due to WDR. $X_{\mathrm{R}} \sim \mathcal{N}\left(0, \sigma_{\mathrm{R}}^{2}\right)$ is a random variable introduced due to WDR.

2) PDP for NLOS Path: The effect of wind velocity and WDR are more dominant on the NLOS path as compared to LOS due to long propagation path, absence of dominant power component, and more scattered energy distribution. Let $P^{\mathrm{LOS}}(n)$ represents the PDP for LOS and $P^{\mathrm{NLOS}}(n)$ is the PDP for NLOS; then we have

$$
\begin{aligned}
P^{\mathrm{LOS}}(n) & =\left\{\begin{array}{ll}
B_{0} & \text { if } \forall \Gamma_{i}, i=1 \\
P^{\mathrm{NLOS}}(n) & \text { if } \forall \Gamma_{i}, i \neq 1
\end{array},\right. \\
P^{\mathrm{NLOS}}(n) & =P^{\mathrm{LOS}}(n)-B_{0},
\end{aligned},
$$

where $B_{0}$ represents the cluster energy due to LOS component. This component is absent in the NLOS case, where the energy is distributed into multiple smaller energy clusters without a dominant energy cluster.

\section{Large and Small Scale Fading Parameters}

We consider that the transmitter and the receivers are static, and only wind velocity and WDR are the main sources of variation in the channel. Large scale fading is considered as a measure of attenuation in the received energy during hurricane conditions as compared to a reference energy. From the empirical data, the empirical attenuation due to wind and WDR can be written as

$$
A_{\mathrm{e}}(v)=10 \log _{10} \frac{\sum_{\forall n} P^{v_{0}}[n]}{\sum_{\forall n} P^{v}[n]},
$$


TABLE I

LARGE SCALE FADING PARAMETERS.

\begin{tabular}{|c|c|c|c|c|c|c|}
\hline Param. & P1, S1 & P1, S2 & P2, S1 & P2, S2 & P3, S1 & P3, S2 \\
\hline$\alpha$ & 0.182 & 0.122 & 0.15 & 0.06 & -0.01 & -0.005 \\
\hline$A_{\mathrm{w} 0}(\mathrm{~dB})$ & -11.7 & -1.9 & -5.5 & 9.73 & 23 & 25 \\
\hline$\sigma_{\mathrm{A}}(\mathrm{dB})$ & 12.39 & 10.09 & 13 & 11.53 & 18.32 & 16.75 \\
\hline
\end{tabular}

TABLE II

SMALL SCALE FADING PARAMETERS.

\begin{tabular}{|c|c|c|c|c|c|c|}
\hline Param. & P1, S1 & P1, S2 & P2, S1 & P2, S2 & P3, S1 & P3, S2 \\
\hline$\mu_{\mathrm{mf}}(\mathrm{dB})$ & -2.69 & -2.96 & -2.47 & -3.25 & -2.55 & -2.92 \\
\hline$\sigma_{\mathrm{mf}}(\mathrm{dB})$ & -25.2 & -25.7 & -27.2 & -31.5 & -20.5 & -19.9 \\
\hline$\mu_{\mathrm{sc}}(\mathrm{dB})$ & 0.92 & 2.28 & 1.29 & -3.07 & -9.21 & -10.6 \\
\hline$\sigma_{\mathrm{sc}}(\mathrm{dB})$ & 1.21 & 6.26 & -1.75 & -3.85 & -16.5 & -27 \\
\hline
\end{tabular}

where $P^{v}[n]$ is the PDP at distance $d$ and wind velocity $v$ for a discrete sampling instance $n$ of a scan. On the other hand, $P^{v_{0}}[n]$ is the PDP at sampling instance $n$ of a scan at $d=12$ $\mathrm{m}$, wind velocity $v_{0}$ of $1.86 \mathrm{mph}$, ambient temperature of $25^{\circ} \mathrm{C}$ and standard air pressure of $10.135 \mathrm{~N} / \mathrm{cm}^{2}$. The $\sum_{\forall n} P^{v}[n]$ represents total energy for different scans in each scenario for wind velocity $v$, while $\sum_{\forall n} P^{v_{0}}[n]$ represents total energy for different scans of the reference scenario.

We use linear regression to obtain large scale fading parameters for LOS, NLOS paths as follows

$$
A_{\mathrm{w}}(v)=A_{\mathrm{w}_{0}}+\alpha v+X_{\mathrm{A}},
$$

where $A_{\mathrm{w}}(v)$ is the attenuation as a function of wind velocity in the presence and absence of rain at a given distance $d$ between the transmitter and the receiver, $A_{\mathrm{w}_{0}}$ is the regression constant, and $\alpha$ is the slope of the linear regression, which depends on WDR and pressure head, $X_{\mathrm{A}}$ is a random variable representing the variations as noise in $A_{\mathrm{w}}(v)$ given as $X_{\mathrm{A}} \sim \mathcal{N}\left(0, \sigma_{\mathrm{A}}^{2}\right)$. The large scale fading parameters that fits in to the model developed in this section using the empirical data explained in Section IV are given in Table I.

Small scale fading for UWB signals in hurricane is due time dispersion of MPCs. There is no Doppler spread present due to static position of the transmitter and the receivers. The small scale fading amplitudes are better fitted with Nakagami distribution given as

$$
F(y ; m, \Omega)=\frac{2 m^{m} y^{2 m-1}}{\Gamma(m) \Omega^{m}} \exp \left(\frac{-m y^{2}}{\Omega}\right),
$$

where $m$ is the shape factor for Nakagami distribution, $\Omega$ represents the spread controlling factor, and $\Gamma(m)$ represents the Gamma function. If $W$ represents the random variable $W \sim \operatorname{Nakagami}(m, \Omega)$, then, we have [17], $m=\frac{E^{2}\left[W^{2}\right]}{\operatorname{Var}\left[W^{2}\right]}$, $\Omega=E\left[W^{2}\right]$, where the Nakagami- $m$ factor is log-normally distributed. Mean and variance of Nakagami- $m$ factor are represented by $\mu_{\mathrm{mf}}$ and $\sigma_{\mathrm{mf}}$, respectively, while the mean and variance of spread controlling factor $\Omega$ are represented by $\mu_{\mathrm{sc}}$ and $\sigma_{\mathrm{sc}}$ respectively. The small scale fading parameters obtained from empirical results that fit the model described above are provided in Table II.

In case of LOS, the Nakagami- $m$ distribution can be approximated with a Rician distribution [18] given as

$$
m \cong \frac{(K+1)^{2}}{2 K+1}, \quad G(y)=\frac{y}{A_{0}} e^{\frac{y^{2}+x^{2}}{2 A_{0}} I_{0} \frac{y x}{A_{0}}},
$$

where $K=10 \log _{10}\left(\frac{x^{2}}{2 A_{0}}\right)$, is the Rician $K$-factor representing the ratio between power of LOS component to the scattered components, $G(y)$ represents the Rician distribution with $A_{0}$ representing the power in the diffuse MPCs, $x^{2}$ is the power in the dominant LOS component, and $I_{0}$ is the $0^{\text {th }}$ order Bessel function. The power in the diffuse component $A_{0}$ in the hurricane conditions can be represented as

$$
A_{0}=A_{\mathrm{b}}\left(c_{\mathrm{r} 0} b_{\mathrm{r} 0}+c_{\mathrm{p} 0} b_{\mathrm{p} 0}+c_{\mathrm{w} 0} v_{\mathrm{w} 0}\right)+X_{\mathrm{A}_{0}},
$$

where $A_{\mathrm{b}}$ is the diffuse power of the base case, $c_{\mathrm{r} 0} \geq 0$, $c_{\mathrm{w} 0} \geq 0$ are constants proportional to WDR, and wind velocities respectively, $c_{\mathrm{p} 0} \geq 0$, is a constant that has higher value for lower pressure head regions and vice versa, $b_{\mathrm{r} 0}, b_{\mathrm{p} 0}$, and $v_{\mathrm{w} 0}$ are the coefficient values for respective constants, where $b_{\mathrm{r} 0}=[0,1]$ and $b_{\mathrm{p} 0}=[0,1]$, representing either absence or presence of rain and lower pressure head respectively, and $v_{\mathrm{w} 0}=$ [90:10:140] in our case, and $X_{\mathrm{A}_{0}} \sim \mathcal{N}\left(0, \sigma_{\mathrm{A} 0}^{2}\right)$ is a Gaussian distributed random variable. We use linear least square error to calculate the values of the constants and distribution of $X_{\mathrm{A}_{0}}$ in $(13)$ as follows:

$$
\left[\begin{array}{lll}
c_{\mathrm{r} 0} & c_{\mathrm{p} 0} & c_{\mathrm{w} 0}
\end{array}\right]^{\mathrm{T}}=\sum_{i i=1}^{24} \sum_{j j=1}^{3}\left(M_{i i, j j}^{\mathrm{T}} M_{i i, j j}\right)^{-1} M_{i i, j j}^{\mathrm{T}}\left(A_{0}-A_{\mathrm{b}}\right),
$$

where $M$ is a matrix that contains the values $\left[\begin{array}{lll}b_{\mathrm{r} 0} & b_{\mathrm{p} 0} & v_{\mathrm{w} 0}\end{array}\right]$.

The variation of power for diffuse and direct components in different scenarios averaged over scans is represented as $X_{\mathrm{Df}} \sim \mathcal{N}\left(\mu_{\mathrm{Df}}, \sigma_{\mathrm{Df}}^{2}\right)$ and $X_{\mathrm{Dr}} \sim \mathcal{N}\left(\mu_{\mathrm{Dr}}, \sigma_{\mathrm{Dr}}^{2}\right)$ respectively. The variations in $K$-factor is represented by $X_{\mathrm{K}} \sim$ $\mathcal{N}\left(\mu_{\mathrm{K}}, \sigma_{\mathrm{K}}^{2}\right)$. In case of NLOS, $K=0$ and the Rician distribution converges to Rayleigh distribution.

\section{EMPIRICAL RESULTS}

This section provides an analysis of large and small scale fading, and the statistics of the MPCs, based on the experiments described in Section II. After collecting the data, post processing of the measurement data is carried out in Matlab.

\section{A. Large and Small Scale Fading Analysis}

Large scale attenuation in our case is a measure of reduction in the received energy during hurricane conditions. Large scale fading for different radio positions and respective scenarios as a function of wind velocity, based on (9) and (10), is shown in Fig. 5 Results show that in general, higher attenuation is observed at higher wind speeds for LOS scenarios, while for NLOS scenarios no critical impact of wind speed is observed. Comparison of our results with related literature [4], [5], [8] indicates that we observe higher attenuation in our experiment, which may be due to following reasons.

First, change in the ambient conditions under different wind velocities can lead to attenuation without rain. This is due to ambient noise that varies with wind flows and pressure heads. At P2, we have lower pressure head that introduces higher wind gusts. Also, at P2, we observe bouncing and scattering of strong wind currents from the obstacle. This results in additional ambient noise at $\mathrm{P} 2$ due to exposure to more agitated molecules resulting in more attenuation. Second, the mechanical turbulences of communication, tie down and relaying equipment introduces variations in the received 


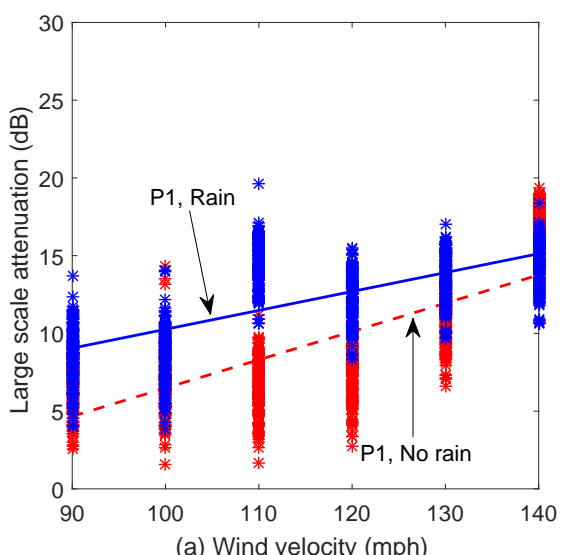

(a) Wind velocity (mph)

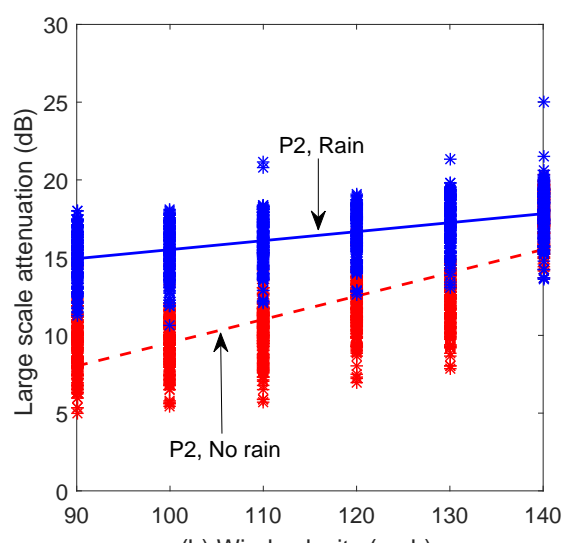

(b) Wind velocity (mph)

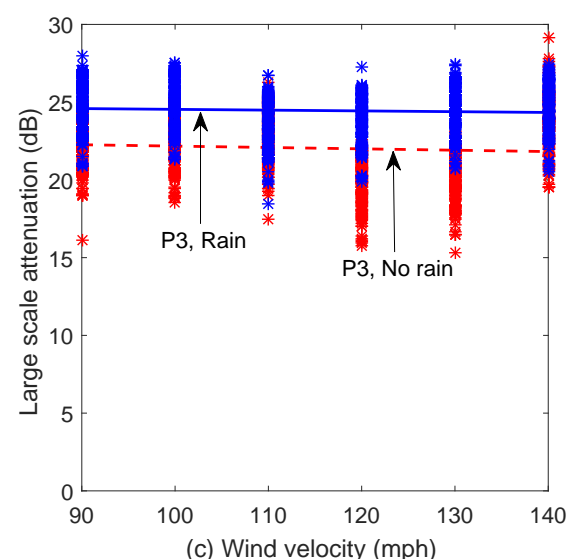

(c) Wind velocity (mph)

Fig. 5. Large scale attenuation at different wind velocities for radio positions (a) P1 (LOS), (b) P2 (LOS), (c) P3 (NLOS).

power. The highest mechanical turbulences are experienced by the exposed antennas. With transmitter and receivers at same height, we observe additional attenuation due to non-alignment of the bore-sight of antennas [13]. Another reason is the limitation of the communication equipment itself. For a given receiver sensitivity, a reduction in the SNR due to ambient noise and mechanical turbulences during hurricane conditions causes the packets to be dropped at the receiver. This implies a more lossy channel. Finally, in case of WDR, the wind intensity we considered is much higher than considered in the literature [4], [8]. This high intensity rain driven by high wind velocities form a kind of water wall between the transmitter and the receiver. Due to higher dielectric constant and loss tangent of the water as compared to rain, we observe additional attenuation during rain. This attenuation is accompanied by scattering, refraction and diffraction of EM waves and higher ambient noise from water droplets that depends on the droplet size distribution. Additionally, the accumulation of water on different surrounding objects result in reduced reflections of incident EM energy due to absorption.

In case of NLOS measurements in Fig. 5(c), we have higher attenuation as the energy is compared with the standard LOS path scenario. In addition to the effects explained above, the absence of dominant LOS component results in more attenuation as compared to LOS.

The probability density function (PDF) of small scale fading amplitudes at different radio positions and respective scenarios is shown in Fig. 6 for wind velocity of $90 \mathrm{~m} / \mathrm{s}$. A general trend is that with WDR, there is decrease of mean and variance of the PDF for both LOS and NLOS paths. This reduction is higher for P2 as compared to P1 for the LOS path. We can deduce that with rain and low pressure head, we expect more attenuation and lower small scale amplitude fluctuations. The mean and variance of the LOS path is greater than the NLOS path. This is validated by the Nakagami- $m$ factor and spread controlling factor $\Omega$ values given in Table II. It can be observed that for NLOS, the distribution fits closely to Raleigh. Similar results are obtained for other wind velocities.

\section{B. Multipath Channel Analysis}

The MPCs from empirical data are analyzed to determine the behavior of the channel and validate the stochastic model

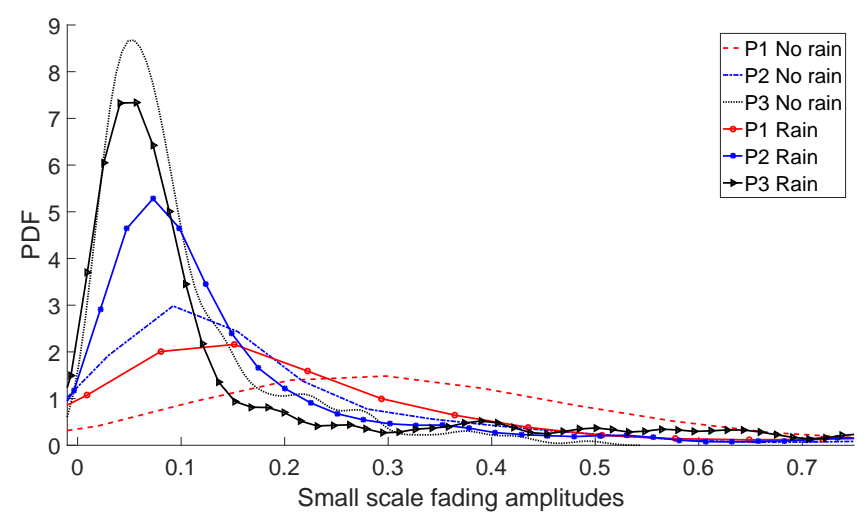

Fig. 6. Probability density function of small scale fading amplitudes for wind velocity $v=90 \mathrm{mph}$ for different radio positions and respective scenarios.

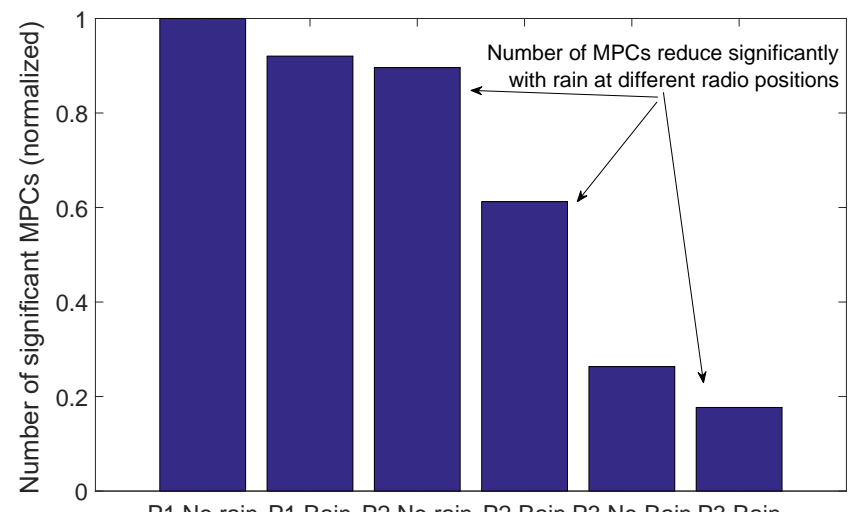

Fig. 7. Number of significant MPCs above threshold averaged over scans for different radio positions and corresponding scenarios (normalized to the number of significant MPCs for no rain scenario at P1).

developed in Section IV. Significant MPCs are characterized to have amplitude greater than the threshold set at $15 \%$ of the maximum amplitude for a given scenario as shown in Fig. 77. It can be observed that number of significant MPCs are affected by radio position and rain in case of LOS due to the effects discussed earlier resulting in weakening of MPC amplitudes 


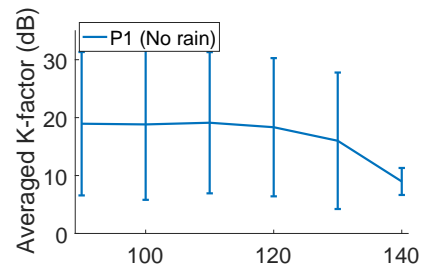

(a) Wind velocity ( $\mathrm{mph}$ )

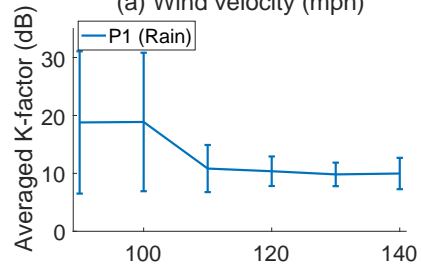

(c) Wind velocity (mph)

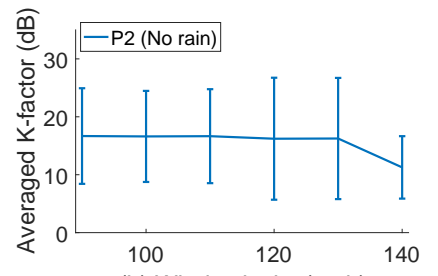

(b) Wind velocity (mph)

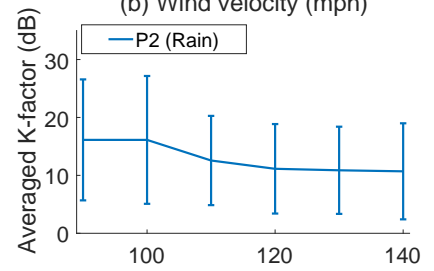

(d) Wind velocity (mph)

Fig. 8. $K$-factors for different wind velocities at different radio positions (a) P1 (no rain), (b) P2 (no rain), (c) P1 (rain), (d) P2 (rain).

especially during rain. In case of NLOS, we observe higher reduction as the arriving MPCs are weak. The multipath statistical channel parameters obtained from empirical data, that fit the model for MPCs propagation through hurricanes, developed in Section III are given in Table III.

The Rician $K$-factor plot for the LOS path averaged over multiple scans is shown in Fig. 8. From (13), a decrease in $K$-factor is observed with the increase in the wind velocities similar to [5] and [9], especially in case of WDR. It can be observed that there is less effect of pressure head on the $K$-factor without rain, as the dominant LOS component is less affected by wind velocities as shown in Fig. 8(a) and Fig. 8(b). The $K$-factor remains almost constant and changes significantly only at $140 \mathrm{mph}$ indicating that ratio of dominant LOS component power and diffuse components power remains proportional except at $140 \mathrm{mph}$ where the dominant LOS component power has reduced significantly. In case of WDR shown in Fig. 8(c) and Fig. 8(d), we observe a significant reduction in the $K$-factor at $110 \mathrm{mph}$ indicating that due to rain the dominant LOS component is reduced earlier as compared to no rain. For the rest of the wind velocities, the $K$-factor remains same indicating that there is proportional reduction in the dominant LOS component and diffuse component.

\section{COnClusions And Future Work}

In this work, we have conducted UWB propagation channel measurements in different hurricane conditions. The effects of wind velocity and WDR at different pressure head regions are monitored in LOS and NLOS communication paths. Based on the measurements, a UWB channel model for hurricanes is developed. It is observed that UWB communications under different wind velocities, pressure heads and WDR for LOS and NLOS paths in a hurricane introduce different effects on the channel model parameters (large scale, small scale and MPCs propagation). The proposed model can help in improved design of communications systems in hurricane conditions. Our future work includes developing channel models in hurricane conditions for millimeter wave communications where more severe attenuation is expected to occur.

TABLE III

CHANNEL MODEL PARAMETERS.

\begin{tabular}{|c|c|c|c|c|c|c|}
\hline Param. & P1, S1 & P1, S2 & P2, S1 & P2, S2 & P3, S1 & P3, S2 \\
\hline $\bar{N}$ & 5.2 & 4 & 3.33 & 3.1 & 1.67 & 1.67 \\
\hline$\gamma(1 / \mathrm{ns})$ & 0.11 & 0.06 & 0.043 & 0.027 & 0.017 & 0.017 \\
\hline$\zeta(1 / \mathrm{ns})$ & 16.32 & 12.6 & 9.32 & 5.61 & 2.33 & 0.3 \\
\hline$\Lambda(\mathrm{ns})$ & 2.3 & 2.45 & 2.38 & 2.47 & 1.72 & 1.86 \\
\hline$\lambda(\mathrm{ns})$ & 0.8 & 0.91 & 0.82 & 0.85 & 0.54 & 0.61 \\
\hline$\sigma_{\mathrm{a}}(\mathrm{dB})$ & 23.14 & 21.22 & 21.56 & 18.51 & 15.12 & 13.2 \\
\hline$\sigma_{\overline{\mathrm{N}}}$ & 1.59 & .98 & .836 & .837 & .53 & .516 \\
\hline$\sigma_{\mathrm{c}}(\mathrm{ns})$ & 16.4 & 34.5 & 43.93 & 49.47 & 51.63 & 52.14 \\
\hline$\sigma_{\mathrm{m}}(n s)$ & 0.287 & 0.268 & 0.277 & 0.265 & 0.28 & 0.253 \\
\hline$\sigma_{\mathrm{P}}(\mathrm{dB})$ & 21.3 & 20.1 & 20.03 & 19.72 & 17.41 & 14.1 \\
\hline$\sigma_{\mathrm{mp}}(\mathrm{dB})$ & 55.14 & 38.44 & 33.22 & 26.77 & 20.17 & 15.22 \\
\hline$\sigma_{\mathrm{R}}(\mathrm{dB})$ & - & 5.77 & - & 6.39 & - & 7.31 \\
\hline$\sigma_{\mathrm{A} 0}(\mathrm{~dB})$ & 28.97 & 10.45 & 8.44 & .219 & 6.46 & .959 \\
\hline$\sigma_{\mathrm{Df}}(\mathrm{dB})$ & 18.83 & 17.79 & 17.6 & 15.3 & 12.78 & 11.45 \\
\hline$\mu_{\mathrm{Df}}(\mathrm{dB})$ & 16.19 & 15.17 & 15.28 & 13.54 & 11.78 & 10.9 \\
\hline$\sigma_{\mathrm{Dr}}(\mathrm{dB})$ & 11.53 & 10.97 & 10.64 & 9.6 & - & - \\
\hline$\mu_{\mathrm{Dr}}(\mathrm{dB})$ & 33.3 & 31 & 30.7 & 27.86 & - & - \\
\hline$\sigma_{\mathrm{K}}(\mathrm{dB})$ & 14.64 & 14.5 & 11.2 & 6.44 & - & - \\
\hline$\mu_{\mathrm{K}}(\mathrm{dB})$ & 17.69 & 14.43 & 16 & 13.79 & - & - \\
\hline
\end{tabular}

\section{REFERENCES}

[1] J. S. Blake. (2005) National hurricane center. [Online]. Available: http://www.nhc.noaa.gov/pastdec.shtml

[2] J. Foerster and Q. Li, "UWB channel modeling contribution form Intel," IEEE 802.15. 3 Wireless personal Area Network, Tech. Rep. IEEE p802. 15-02/279rO-SG3a, 2002.

[3] A. F. Molisch, K. Balakrishnan, C.-C. Chong, S. Emami, A. Fort, J. Karedal, J. Kunisch, H. Schantz, U. Schuster, and K. Siwiak, "IEEE 802.15. 4a channel model-final report," IEEE P802, vol. 15, no. 04, p. $0662,2004$.

[4] Y. S. Meng, Y. H. Lee, and B. C. Ng, "The effects of tropical weather on radio-wave propagation over foliage channel," IEEE Trans. Vehic. Technol., vol. 58, no. 8, pp. 4023-4030, Oct 2009.

[5] D. Crosby, V. S. Abhayawardhana, I. J. Wassell, M. G. Brown, and M. P. Sellars, "Time variability of the foliated fixed wireless access channel at $3.5 \mathrm{GHz}$," in Proc. IEEE Vehic. Technol. Conf., vol. 1, May 2005, pp. 106-110 Vol. 1.

[6] E. R. Pelet, J. E. Salt, and G. Wells, "Effect of wind on foliage obstructed line-of-sight channel at $2.5 \mathrm{GHz}$," IEEE Trans. Broadcasting, vol. 50 , no. 3, pp. 224-232, Sept. 2004.

[7] S. Perras and L. Bouchard, "Fading characteristics of RF signals due to foliage in frequency bands from 2 to $60 \mathrm{GHz}$," in Proc. IEEE Int. Symp., 2002., vol. 1, 2002, pp. 267-271.

[8] S.-H. Fang and Y.-H. S. Yang, "The impact of weather condition on radio-based distance estimation: A case study in GSM networks with mobile measurements," IEEE Trans. Vehic. Technol., vol. 65, no. 8, pp. 6444-6453, 2016.

[9] M. H. Hashim and S. Stavrou, "Measurements and modelling of wind influence on radiowave propagation through vegetation," IEEE Trans. Wireless Commun., vol. 5, no. 5, pp. 1055-1064, 2006.

[10] E. Simiu, P. Vickery, and A. Kareem, "Relation between Saffir-Simpson hurricane scale wind speeds and peak 3-s gust speeds over open terrain," J. Structural Eng., vol. 133, no. 7, pp. 1043-1045, 2007.

[11] FCC, "First report and order 02-48," Apr. 2002.

[12] I. Guvenc, S. Gezici, Z. Sahinoglu, and U. C. Kozat, Reliable communications for short-range wireless systems. Cambridge University Press, 2011.

[13] Time domain website, 2011. [Online]. Available: http://www. timedomain.com/datasheets/TD_Broadspec_Antenna.pdf

[14] C. G. David Pnueli, Fluid Mechanics. Cambridge University Press, 1992.

[15] R. Medhurst, "Rainfall attenuation of centimeter waves: Comparison of theory and measurement," IEEE Trans. Ant. Propag., vol. 13, no. 4, pp. $550-564,1965$.

[16] A. Saleh and R. Valenzuela, "A statistical model for indoor multipath propagation," IEEE J. Sel. Areas Commun. (JSAC), vol. 5, no. 2, pp. 128-137, Feb. 1987.

[17] R. Kolar, R. Jirik, and J. Jan, "Estimator comparison of the nakagami-m parameter and its application in echocardiography," Radioengineering, vol. 13, no. 1, pp. 8-12, 2004.

[18] J. Paul. (2010) JPL Wireless Comm. [Online]. Available: http://www. wirelesscommunication.nl/reference/chaptr03/ricenaka/ricenaka.htm 